\title{
Optically Triggered Target of Opportunity Observations with the MAGIC Telescope
}

\author{
Elina J. Lindfors ${ }^{* \dagger}$ \\ Tuorla Observatory, University of Turku \\ E-mail: elilin@utu.fi
}

\section{Daniel Mazin}

Institut de Fisica d'Altes Energies (IFAE), Barcelona, Spain

E-mail: mazineifae.es

\section{Kari Nilsson}

Tuorla Observatory, University of Turku

E-mail: kani@utu.fi

\section{Leo Takalo}

Tuorla Observatory, University of Turku

E-mail: takalo@utu.fi

\begin{abstract}
The MAGIC collaboration has been performing Target of Opportunity observations of blazars, when seen flaring in optical by Tuorla blazar monitoring program. In past three years, there has been 5 optical alerts during good weather conditions and reasonable declination of the source. Three alerts have resulted in discovery of VHE gamma-rays from previously undetected source. We will introduce the Tuorla Blazar monitoring program and discuss the successful ToO observations.
\end{abstract}

Workshop on Blazar Variability across the Electromagnetic Spectrum

April 22-25 2008

Palaiseau, France

\footnotetext{
${ }^{*}$ Speaker.

${ }^{\dagger}$ on behalf of the MAGIC Collaboration
} 


\section{Introduction}

MAGIC is an Imaging Air Cerenkov Telescope (IACT) located on La Palma, Canary Islands. Due to its large collecting area and uniquely designed camera MAGIC has reached a lower energy threshold (trigger threshold 50-60 GeV) for gamma-ray emission than any existing terrestial imaging gamma-ray telescope.

Blazars are the most extreme types of Active Galactic Nuclei (AGN). In these objects the dominant radiation component originates in a relativistic jet pointed nearly towards the observer. Blazars show variable flux in all wave bands from radio to very high energy (VHE, defined as E $>100 \mathrm{GeV}$ ) $\gamma$-rays. The relationship between the variability at different wave bands appears rather complicated. The MAGIC collaboration is performing Target of Opportunity observations of sources in high flux state in the optical and/or X-ray band. Alerts of optical high state originate from Tuorla Blazar Monitoring Program. Optically triggered observations have resulted in discovery of VHE $\gamma$-rays from Mrk 180 [1] and 1ES 1011+496 [2]. During the workshop we triggered observations of S5 $0716+714$ which resulted in the discovery of VHE $\gamma$-rays from S5 0716+714 [3] . In this paper we describe the Tuorla Blazar monitoring program and present results of the optically triggered ToO observations with the MAGIC telescope.

\section{Tuorla Blazar Monitoring Program}

The optical monitoring observations are performed with the KVA $35 \mathrm{~cm}$ telescope, which is remotely operated from Finland and the Tuorla $1 \mathrm{~m}$ telescope. The observations are done in the R-band and the magnitudes are measured using differential photometry with calibration stars in the same CCD frame as the object. The light curves are updated to project web page http://users.utu.fi/kani/1m/ on daily basis.

The long term monitoring program consists of 24 objects, which were chosen from a list of candidate TeV blazars by Costamante \& Ghisellini (2002)[4]. The monitoring started in 2002 with the goal to study the optical variability of these sources and to determine the baseline flux. We have also made a study of the host galaxy contribution to the objects overall brightness [5]. This way we can get direct measurement of the AGN core brightness by subtracting the host galaxy flux. The collected lightcurves have been used to study the variability characteristics of these blazars [6] and to define the trigger criteria for Target-of-Opportunity program. As the ToO program has proven to be successful, we have added $\sim 20$ more blazars to the monitoring program.

\section{Optically triggered Target-of-Opportunity observations with MAGIC}

Optically triggered ToO observations have resulted in discovery of three new Very High Energy $\gamma$-ray emitting blazars: Mrk 180 [1], 1ES1011+496 [2] and S5 0716+714 [3].

\subsection{Mrk 180}

Mrk 180 (1ES 1133+704) is a well-known high frequency peaked BL Lac (HBL) at a redshift of $z=0.045$ [7]. In March 2006 it underwent an optical outburst and was observed for 12.4 hours by MAGIC. During these observations VHE $\gamma$-ray emission was detected with a significance of $5.5 \sigma$. 

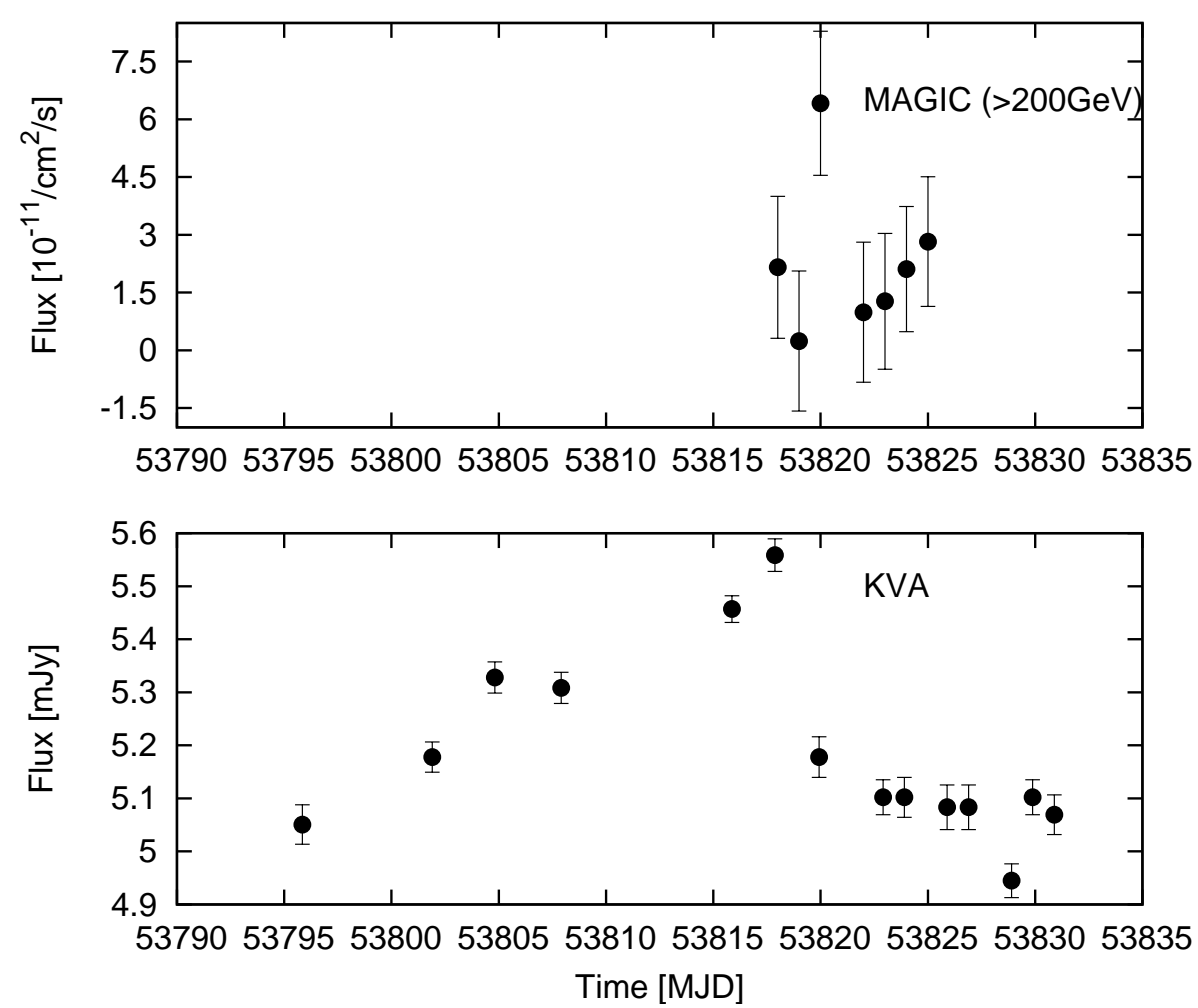

Figure 1: Optical and VHE gamma-ray light curves of Mrk 180 from March 2006.

An integral flux above $200 \mathrm{GeV}$ of $\left(2.3 \pm 0.7 \times 10^{-11}\right) \mathrm{cm}^{-2} \mathrm{~s}^{-1}$ was measured, corresponding to $11 \%$ of the Crab Nebula flux. No significant flux variation was found. Earlier observations by other experiments have only set upper limits on the VHE $\gamma$-ray emission from Mrk 180. The upper limits are above the flux measured by MAGIC. It is therefore not possible to judge whether the detected VHE flux level represents a flaring or a quiescent state of the AGN.

\subsection{ES 1011+496}

1ES $1011+496$ is an HBL object at a redshift of $z=0.212$. The MAGIC observations were triggered by an optical outburst in March 2007 and the source was observed from March to May 2007. The 18.7 hours of data show an excess at $6.2 \sigma$ significance with an integrated flux above $200 \mathrm{GeV}$ of $(1.58 \pm 0.32) \times 10^{-11} \mathrm{~cm}^{-2} \mathrm{~s}^{-1}$. The source has been observed previously by MAGIC in March-April 2006 when the source was in a lower optical state. Those observations only showed weak evidence for a signal at $3.5 \sigma$ [8]. Consequently, the VHE $\gamma$-ray flux was $>40 \%$ higher in March-May 2007 than in March-April 2006, indicating that the VHE emission state may be related to the optical emission state.

\section{$3.3 \mathrm{S5} 0716+714$}

The BL Lac object S5 $0716+714$ has been studied intensively at all frequency bands. It is highly variable with rapid variations observed from radio to X-ray bands [9]. It has therefore been 

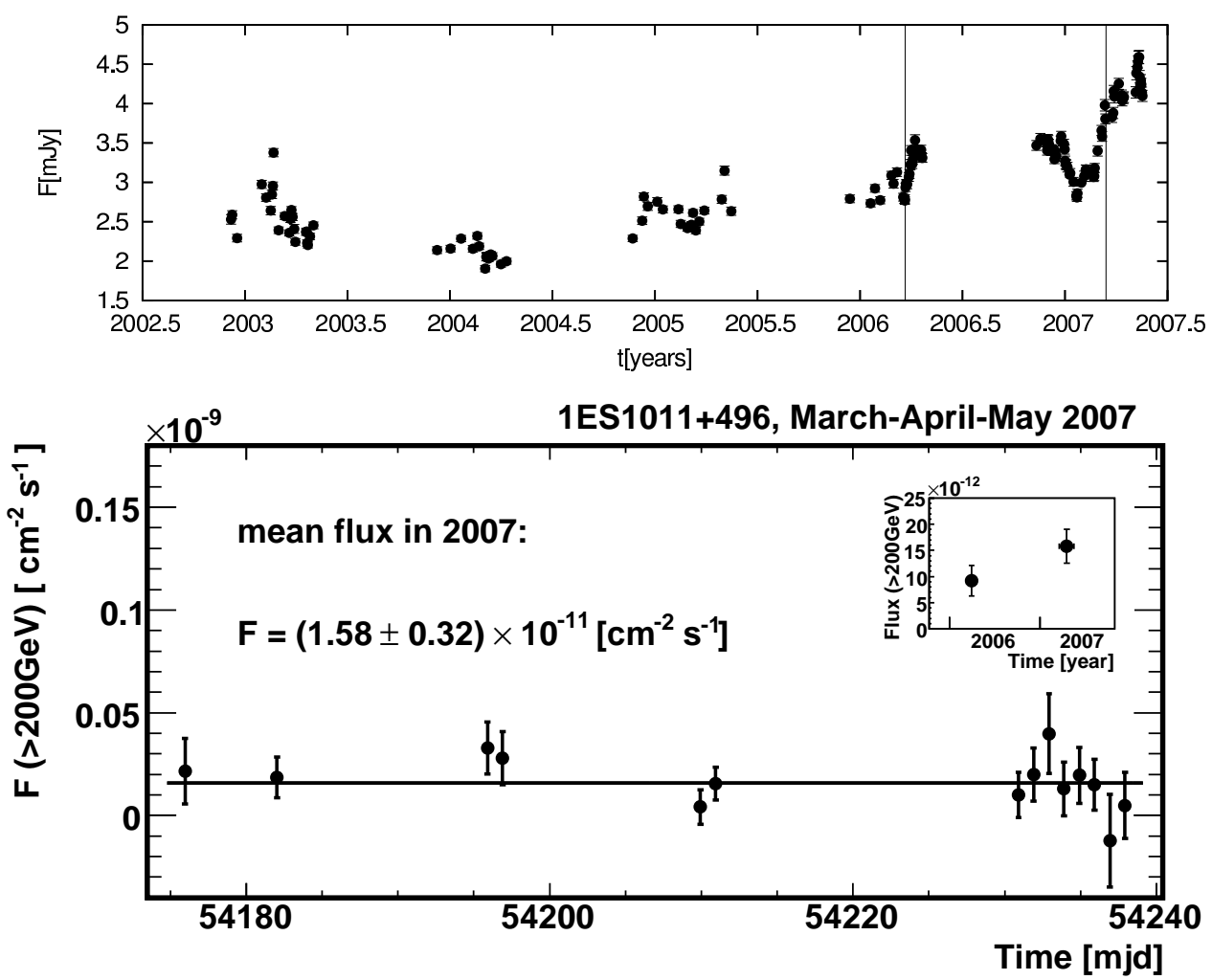

Figure 2: Optical (top) and VHE $\gamma$-ray (bottom) light curves of 1ES 1011+496. The optical lightcurve shows 5 years of data from Tuorla blazar monitoring program and the vertical lines indicate the starting points of the MAGIC observations. The VHE $\gamma$-ray lightcurve shows two months of data from 17th of March to 18th of May 2007. The inset shows the yearly averages.

target to several multiwavelength campaigns, the most recent one organized by GLAST-AGILE Support Program in July-November 2007 [10, 11]. Due to the very bright nucleus which outshines the host galaxy, its redshift is still uncertain. The recent detection of the host galaxy [12] suggests a redshift of $0.31 \pm 0.08$ which is consistent with the redshift $\mathrm{z}=0.26$ determined by spectroscopy for 3 galaxies close to $S 50716+714$ [13, 14]. It can therefore be concluded that $\mathrm{z}=0.26$ seems to be a rather likely redshift for S5 $0716+714$, although with the lack of lines in the optical spectrum $[15,16]$, the ultimate proof for the redshift is still missing. S5 $0716+714$ has a synchrotron peak at optical band and is therefore classified as LBL [17] or IBL [18]. The discovery of S5 0716+714 by MAGIC represents only the third LBL (BL Lac [19]; W Comae [20]) that has been detected at VHE $\gamma$-rays. With a presumable redshift of $\mathrm{z}=0.26$, S5 $0716+714$ is the most distant object of the three and the second most distant of all VHE $\gamma$-ray emitters (after 3C 279 with $\mathrm{z}=0.536$ [21]).

In April 2008 the optical flux of S5 0716+714 almost doubled in three nights (14th of April: 29 mJy, 17th April: $52 \mathrm{mJy}$ ) reaching its historical maximum on April 17th. MAGIC started the observations 5 nights later (22nd), when the moon conditions allowed. The observations resulted in the discovery of VHE $\gamma$-rays from S5 0716+714, announced with an Astronomer Telegram on 30th of April [3]. A detailed paper on the MAGIC results is in preparation. 


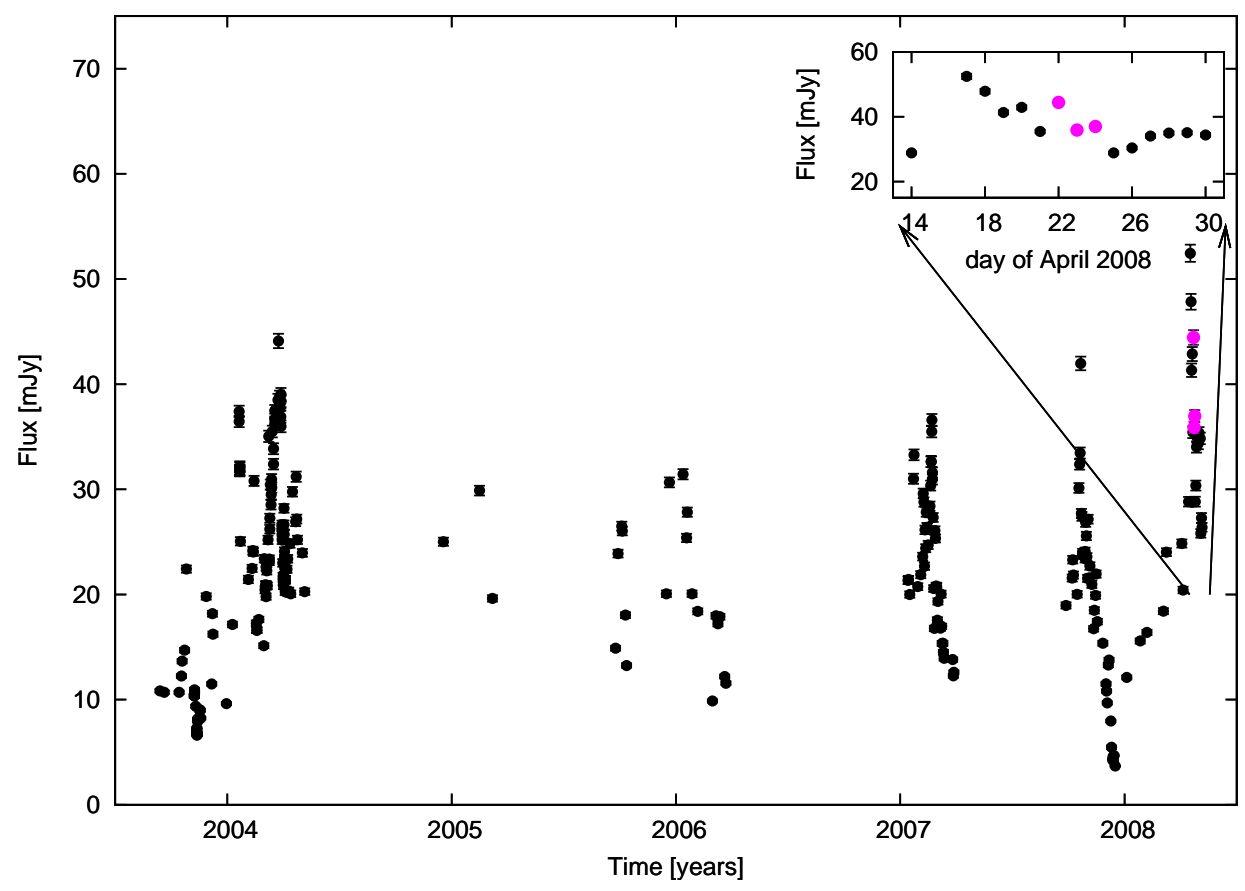

Figure 3: Long term optical lightcurve of S5 0716+714. The inset shows the lightcurve from April 2008, the pink datapoints are simultaneous with MAGIC observations.

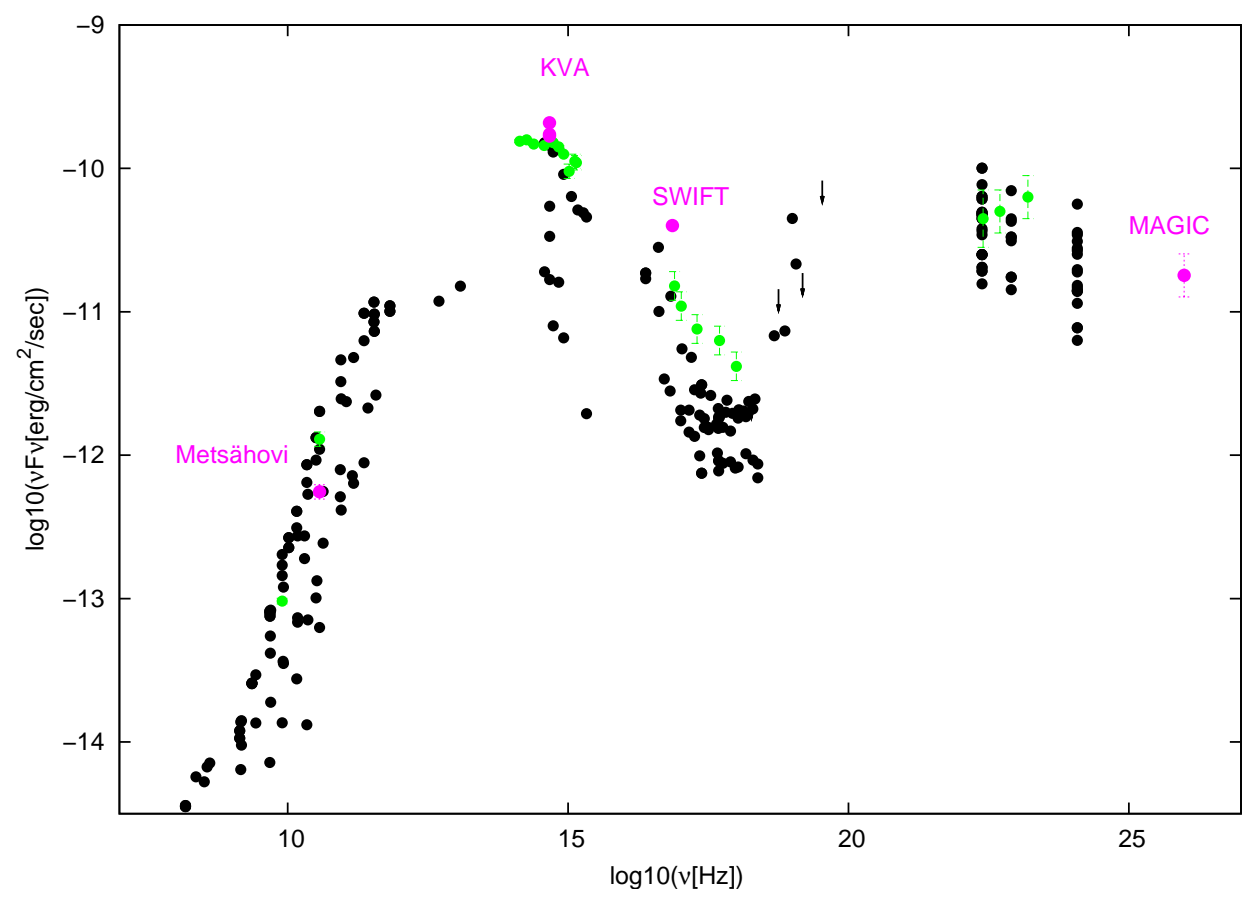

Figure 4: The spectral energy distribution of S5 0716+714. The black data are the historical SED from [22] and the green data points from high state of October 2007 [10, 11]. The pink data points are from April 2008: $37 \mathrm{GHz}$ from Metsähovi radio observatory (courtesy of A. Lähteenmäki), optical from KVA, X-ray from SWIFT [23] and $\gamma$-ray from MAGIC [3] 


\section{Discussion}

MAGIC has performed observations of 5 blazars triggered by their high optical states in 20062008. These ToO observations have resulted in the discovery of three new Very High Energy gamma-ray emitting blazars: Mrk 180 [1], 1ES1011+496 [2] and S5 0716+714 [3]. Additionally to the 5 triggers, part of the BL Lac data in 2005 [19] and the 3C 279 [21] data in 2006, which both resulted in the discovery of VHE gamma-rays from these sources, were taken when the sources were in high optical state. Meanwhile, the MAGIC observations of 1ES 1011+496 and BL Lac during a lower optical state resulted in a weak signal and non-detection, respectively, which seems to indicate that there is a connection between the optical and VHE gamma-ray high states. From two other blazars observed after optical triggers no VHE $\gamma$-rays were detected and a publication on the upper limits for these objects is in preparation. To further study the connection between optical and VHE $\gamma$-ray high states follow-up observations of the discovered sources are needed as well as a continuation of the optically triggered ToO Observations.

\section{References}

[1] Albert, J. et al. (the MAGIC Collaboration) 2006, ApJ, 648, L105

[2] Albert, J. et al. (the MAGIC Collaboration) 2007, ApJ, 666, L17

[3] Teshima, M. et al. (the MAGIC Collaboration) 2008, ATel 1500

[4] Costamante, L. \& Ghisellini, G. 2002, A\&A, 384, 56

[5] Nilsson, K., Pasanen, M., Takalo, L. O. et al. 2007, A\&A, 475, 199

[6] Nilsson, K. et al. 2008, in preparation

[7] Falco, E. E. et al. 1999, PASP, 111, 438

[8] Albert, J. et al. (the MAGIC Collaboration) 2008, ApJ, 681, 944

[9] Wagner, S. J., Witzel, A. Heidt, J. et al. 1996, AJ, 111, 2187

[10] Villata, M. Raiteri, C., Larionov, V. M. et al. 2008, A\&A, 480, 339

[11] Giommi, P., Colafrancesco, S., Cutini, S. et al. 2008a, A\&A, submitted

[12] Nilsson, K., Pursimo, T., Sillanpää, A. et al. 2008, A\&A, accepted

[13] Stickel, M., Fried, J. W. \& Kuehr, H. 1993, A\&AS, 98, 393

[14] Bychova, V. S., Kardashev, N. S., Boldycheva, A. V. et al. 2006, Astronomy Reports, 50, 802

[15] Rector, T. A. \& Stocke, J. T 2001, AJ, 122, 565

[16] Sbarufatti, B., Treves, A., Falomo, R., et al. 2006, AJ, 132, 1

[17] Nieppola, E., Tornikoski, M., Valtaoja, E. 2006, A\&A, 445, 441

[18] Padovani, P., Giommi, P. 1995, MNRAS, 277, 1477

[19] Albert, J. et al. (the MAGIC Collaboration) 2007, ApJ, 667, L21

[20] Swordy, S. (the VERITAS Collaboration) 2008, ATel 1422

[21] Albert, J. et al. (the MAGIC Collaboration) 2008, Science, 320, 1752

[22] Ostorero, L., Wagner, S. J., Gracia, J. et al. 2006, A\&A, 451, 797

[23] Giommi, P., Perri, M., Verrecchia, F. et al. 2008b, ATel 1495 